Y. AIHARA AND J. NOGUCHI

KODAI MATH. J.

14 (1991), 320-334

\title{
VALUE DISTRIBUTION OF MEROMORPHIC MAPPINGS INTO COMPACTIFIED LOCALLY SYMMETRIC SPACES
}

\author{
By Yoshiniro AiHara AND JunJiRo Noguchi
}

\section{Introduction}

There have been many works to generalize the Nevanlinna theory (especially, his second main theorem) to higher dimensional case (cf., e.g., [5], [17], [21]). While the so called equidimensional holomorphic or meromorphic mappings $f: W \rightarrow V$ between algebraic varieties have been well studied, we do not know very much about $f: W \rightarrow V$ with $\operatorname{dim} W<\operatorname{dim} V$ (cf. Noguchi [13], [14] and Siu $[20])$. So far, we have to put some special restriction on target spaces or on the divisors of the target spaces. In this paper, we will establish an inequality of the second main theorem type for meromorphic mappings into a compactification of a locally symmetric space.

Let $\mathscr{D}$ be a bounded symmetric domain in $C^{m}$ and $\Gamma$ a neat arithmetic discrete subgroup of the holomorphic transformation group Aut (D) of $\mathscr{D}$. Let $f: \boldsymbol{C}^{n} \rightarrow \overline{\Gamma \backslash \mathscr{D}}$ be a meromorphic mapping, where $\overline{\Gamma \backslash \mathscr{D}}$ is a smooth toroidal compactification of $\overline{\Gamma \backslash \mathscr{D}}$. Nadel [11] proved that if $\Gamma$ is sufficiently small, then the image $f(\boldsymbol{C})(n=1)$ of any non-constant holomorphic curve $f: \boldsymbol{C} \rightarrow \overline{\Gamma \backslash \mathscr{D}}$ is contained in

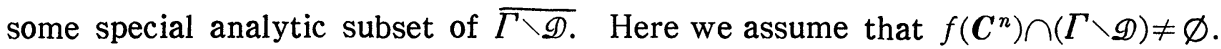
Then, of course, $f$ hits the boundary divisor $D=\overline{\Gamma \backslash \mathscr{D}}-(\Gamma \backslash \mathscr{D})$, since $\Gamma \backslash \mathscr{D}$ is (complete) hyperbolic. We are concerned with how often $f$ hits $D$. We prove the following inequality of the second main theorem type for $f: C^{n} \rightarrow \overline{\Gamma \backslash \mathscr{D}}$ of maximal rank:

$$
\begin{aligned}
& K\left\{T_{f}(r,[D])+T_{f}(r, K(\overline{\Gamma \backslash \mathscr{D})})\}\right. \\
& \leqq N\left(r, \operatorname{Supp} f^{*} D\right)+O(\log r)+O\left(\log ^{+} T_{f}(r,[D])\right) \| .
\end{aligned}
$$

Here $K$ is a positive constant depending only on $\mathscr{D}$ and $n$, Supp $f^{*} D$ is the support of the pull-back divisor $f^{*} D$ and the sign "\|" means as usual in the Nevanlinna theory that the estimate holds as $r \rightarrow \infty$ outside an exceptional subset with finite length (see Theorem (2.2)).

In the Nevanlinna theory for meromorphic functions, one dimensional complex projective space $\boldsymbol{P}^{1}(\boldsymbol{C})$ minus three distinct points $(a, b$ and $c)$ is of a

Received November 6, 1990. 
crucial importance. Note that $\boldsymbol{P}^{1}(\boldsymbol{C})-\{a, b, c\}$ is biholomorphic to $\Gamma(2) \backslash \boldsymbol{H}$. where $\boldsymbol{H}$ is the upper half plane and $\Gamma(2) \subset P S L(2, \boldsymbol{Z})$ is the congruence subgroup of level 2 (cf. $\S 3$ ).

The proof of (i) is based on some current inequalities similar to those in Nadel [11] and Noguchi [16]. We derive from (i) a certain defect relations

$$
\Theta_{f}(D) \leqq \theta_{0}<1,
$$

where $\theta_{0}$ is a constant independent from $f$ (see $\S 3$ ).

In $\S 4$, we discuss the generalizations of the domain space $\boldsymbol{C}^{n}$ of $f$ to an affine algebraic variety and to a finite analytic covering space of $\boldsymbol{C}^{n}$. We also consider a holomorphic mapping $f: \Delta^{*} \rightarrow \overline{\Gamma \backslash \mathscr{D}}$ from the punctured disc $\Delta^{*}$ into $\overline{\Gamma \backslash \mathscr{D}}$. In this case, if $f$ does not hit $D$, then an inequality similar to (i) implies that $f$ extends holomorphically from $\Delta$ into $\overline{\Gamma \backslash \mathscr{D}}$ (cf. Kobayashi-Ochiai [8], Borel [3], Kiernan [6], Kobayashi [7], Kwack [9] and Noguchi [13], [15]).

\section{Preliminaries}

(a) Lemmas on currents. Let $M$ be a paracompact complex manifold of dimension $m$. We set

$$
d^{c}=\frac{\sqrt{-1}}{4 \pi}(\bar{\partial}-\partial)
$$

For a locally integrable function $\varphi$ on $M$, we denote by $d d^{c}[\varphi]$ the $d d^{c}$-derivative in the sense of current.

LEMMA (1.1) (cf., e.g., [5] or [17]). Let $F$ be a holomorphic function on $M$ with zero divisor $(F)$ and $h$ a positive valued $C^{\infty}$-function on $M$. Then

$$
\begin{gathered}
d d^{c}\left[\log |F|^{2}\right]=(F), \\
d d^{c}\left[\log \left(\log \left(|F|^{2} h\right)\right)^{2}\right]=d d^{c} \log \left(\log \left(|F|^{2} h\right)\right)^{2} .
\end{gathered}
$$

Let $D$ be a hypersurface of $M$ with only normal crossings. Let $p_{0} \in D$ and take a holomorphic local coordinate neighborhood system $U\left(w_{1}, \cdots, w_{m}\right)$ around $p_{0}$ so that

$$
\begin{aligned}
& U \cong \Delta^{m}=\left\{\left(w_{\nu}\right) ;\left|w_{\nu}\right|<1\right\} \\
& p_{0}=(0, \cdots, 0) \text { and } U \cap D=\left\{w_{1} \cdots w_{k}=0\right\}
\end{aligned}
$$

where $1 \leqq k \leqq m$. Let $\omega_{0}$ be the following Kähler form on $U-D \cong\left(\Delta^{*}\right)^{k} \times \Delta^{m-k}$ :

$$
\begin{gathered}
\omega_{0}=\sum_{\nu=1}^{k} \frac{1}{\left|w_{\nu}\right|^{2}\left(\log \left|w_{\nu}\right|^{2}\right)^{2}} \frac{\sqrt{-1}}{2 \pi} d w_{\nu} \wedge d \bar{w}_{\nu}+\sum_{\nu=k+1}^{m} \frac{\sqrt{-1}}{2 \pi} d w_{\nu} \wedge d \bar{w}_{\nu} \\
=d d^{c} \varphi_{0},
\end{gathered}
$$


where

$$
\varphi_{0}=-\sum_{\nu=1}^{k} \log \left(\log \left|w_{\nu}\right|^{2}\right)^{2}+\sum_{\nu=k+1}^{m}\left|w_{\nu}\right|^{2}
$$

DEFinition (1.5). We say that a smooth $(1,1)$-form $\omega$ on $M-D$ has at most Poincaré growth near $p_{0}$ if $|\omega|=O\left(\omega_{0}\right)$ on $U$, and that $\omega$ has at most Poincaré growth near $D$ if it has at most Poincaré growth near any point of $D$.

Note that if $\omega$ has at most Poincare growth near $D$, then $\omega$ is locally integrable on $M$.

LEMMA (1.6). Let $M$ and $D$ be as above. Let $\varphi: M-D \rightarrow \boldsymbol{R}$ be a smooth function which is bounded from above. Let $\omega_{1}$ and $\omega_{2}$ be real smooth $(1,1)$-forms on $M-D$ such that $\omega_{1} \geqq 0$ and $\omega_{2}$ has at most Poincaré growth near D. Assume that

$$
d d^{c} \varphi \geqq \omega_{1}+\omega_{2} \quad \text { on } M-D .
$$

Then we have the following:

(i) $\varphi$ is locally written as the difference of two plurisubharmonic functions; i.e., locally on $M, \varphi=\varphi_{1}-\varphi_{2}$ with plurisubharmonic functions $\varphi_{1}$ and $\varphi_{2}$,

(ii) $\omega_{1}$ is locally integrable on $M$,

(iii) $d d^{c}[\varphi] \geqq \omega_{1}+\omega_{2}$ on $M$.

This was implicitly proved in Nadel [11]. Here we give a simplified proof due to Noguchi [16] for a convenience.

Proof. It suffices to show (i) (iii) around a point $p_{0} \in D$. Take a holomorphic local coordinate neighborhood system $U\left(w_{1}, \cdots, w_{m}\right)$ as in (1.2). Let $\varphi_{0}$ be a function on $U-D$ defined by (1.4). Then $\varphi_{0}$ is plurisubharmonic on $U$ and there is a constant $C>0$ such that

Put

$$
C d d^{c} \varphi_{0}+\omega_{2} \geqq 0 .
$$

Then we have

$$
\varphi_{1}=\varphi+C \varphi_{0}
$$

$$
d d^{c} \varphi_{1} \geqq \omega_{1}+\omega_{2}+C d d^{c} \varphi_{0} \geqq \omega_{1} \geqq 0
$$

on $U-D$. Thus $\varphi_{1}$ is plurisubharmonic and bounded from above on $U-D$, so that $\varphi_{1}$ is plurisubharmonic on $U$ (cf., e.g., [17, Chap. III]). As $C \varphi_{0}$ is plurisubharmonic on $U$, (i) is proved. We obtain (ii) also from (1.7). By Lemma (1.1), (ii), we see

$$
d d^{c}\left[\varphi_{1}\right]=d d^{c}[\varphi]+C d d^{c} \varphi_{0} .
$$

Let $d d^{c}\left[\varphi_{1}\right]=\eta_{\mathrm{reg}}+\eta_{\mathrm{sing}}$ be the Lebesgue decomposition into regular and singular 
parts. Then (1.7) implies

so that

$$
\eta_{\mathrm{reg}} \geqq \omega_{1}+\omega_{2}+C d d^{c} \varphi_{0}
$$

$$
d d^{c}[\varphi]+C d d^{c} \varphi_{0}=\eta_{\mathrm{reg}}+\eta_{\mathrm{sing}} \geqq \eta_{\mathrm{reg}} \geqq \omega_{1}+\omega_{2}+C d d^{c} \varphi_{0} .
$$

Hence we deduce (iii). Q.E.D.

(b) First main theorem. Let $z=\left(z_{1}, \cdots, z_{n}\right)$ be the natural complex coordinate system in $\boldsymbol{C}^{n}$ and set

$$
\begin{gathered}
\|z\|^{2}=\sum_{\nu=1}^{n} z_{\nu} \bar{z}_{\nu}, \quad B(r)=\left\{z \in C^{n} ;\|z\|<r\right\} \\
S(r)=\left\{z \in C^{n} ;\|z\|=r\right\}, \quad \alpha=d d^{c}\|z\|^{2}, \\
\eta=d^{c} \log \|z\|^{2} \wedge\left(d d^{c} \log \|z\|^{2}\right)^{n-1}
\end{gathered}
$$

For a $(1,1)$-current $\varphi$ of order 0 on $C^{n}$, we set

$$
\begin{gathered}
n(r, \varphi)=r^{2-2 n}\left\langle\varphi \wedge \alpha^{n-1}, \chi_{B(r)}\right\rangle, \\
N(r, \varphi)=\int_{1}^{r} \frac{n(t, \varphi)}{t} d t,
\end{gathered}
$$

where $\chi_{B(r)}$ denotes the characteristic function of $B(r)$. We recall well-known Jensen's formula (cf. [19] for a proof).

LEMMA (1.9). Let $u$ be a function on $\boldsymbol{C}^{n}$ such that $u$ is locally written as the difference of two plurisubharmonic functions. Then

$$
\int_{S(R)} u \eta-\int_{S(r)} u \eta=2 \int_{r}^{R} n\left(t, d d^{c}[u]\right) \frac{d t}{t}
$$

for $R>r>0$.

Let $N$ be a compact complex manifold and $f: \boldsymbol{C}^{n} \rightarrow N$ a meromorphic mapping. Let $\omega$ be a real $(1,1)$-form on $N$ such that the pull-back $f^{*} \omega$ of $\omega$ by $f$ is locally integrable. Then we set

$$
T_{f}(r, \omega)=N\left(r, f^{*} \omega\right)
$$

and call it the characteristic function of $f$ with respect to $\omega$.

Let $L \rightarrow N$ be a holomorphic line bundle over $N$ with a hermitian fiber metric $|\cdot|$ and $\omega$ its Chern form. We set

$$
T_{f}(r, L)=T_{f}(r, \omega) .
$$

Let $|L|$ denote the complete linear system of $L$ and $D \in|L|$. Choose a global holomorphic section $\sigma \in H^{\circ}(N, L)$ so that the divisor $(\sigma)$ defined by $\sigma$ is $D$ and $|\sigma|<1$. Let Supp $D$ be the support of $D$. Assume that $f\left(\boldsymbol{C}^{n}\right) \subset \operatorname{Supp} D$. Set 


$$
m_{f}(r, D)=-\int_{S(r)} \log f^{*}|\sigma| \eta \geqq 0
$$

THEOREM (1.10). Let the notation be as above. Then we have the following.

(i) (First Main Theorem)

$$
T_{f}(r, L)=N\left(r, f^{*} D\right)+m_{f}(r, D)+O(1),
$$

where $O(1)$ stands for a bounded term as $r \rightarrow+\infty$.

(ii) (Nevanlinna Inequality)

$$
N\left(r, f^{*} D\right) \leqq T_{f}(r, L)+O(1) .
$$

For the proof, we see by Lemma (1.1) that

$$
d d^{c}\left[\log \left|f^{*} \sigma\right|^{2}\right]=f^{*} D-f^{*} \omega .
$$

In the next section, we deal with the case where $M$ is Moishezon and $M-D$ is quasi-projective; in this case, we deduce Theorem (1.10) from Lemma (1.9) and (1.11). For the general case, see Stoll [21].

An inequality of the second main theorem type is, in short, an estimate opposite to (ii); that is, it provides a bound of $T_{f}(r, L)$ in terms of $N\left(r, f^{*} D\right)$. We define the defect $\Theta_{f}(D)$ of $D$ by

$$
\Theta_{f}(D)=1-\limsup _{r \rightarrow+\infty} \frac{N\left(r, \operatorname{Supp} f^{*} D\right)}{T_{f}(r, L)} .
$$

It is clear that $0 \leqq \Theta_{f}(D) \leqq 1$, and that if $f\left(C^{n}\right) \cap \operatorname{Supp} D=\varnothing$, then $\Theta_{f}(D)=1$.

\section{Inequality of the second main theorem type}

Let $\mathscr{D}$ be a bounded symmetric domain in $\boldsymbol{C}^{m}$ and $g$ the Bergman metric on $\mathscr{D}$ normalized so that the Ricci curvature

$$
\operatorname{Ricci}(g)=-1 \text {. }
$$

We denote by $H(X, Y)$ the holomorphic bisectional curvature of $g$. Then it is known that $H(X, Y) \leqq 0$ and

$$
H(X, X) \leqq-\gamma
$$

for some number $\gamma$ such that $1 / m \leqq \gamma \leqq 1$ (cf., e.g., [2, p. 219]).

Let $\Gamma \subset$ Aut $(\mathscr{D})$ be a neat arithmetric discrete subgroup ([1]). Then $V=$ $\Gamma \backslash \mathscr{D}$ is a smooth quasi-projective algebraic variety. Let $\bar{V}$ be a smooth toroidal compactification of $V$ such that $D=\bar{V}-V$ is a hypersurface with only normal crossings $([1],[10])$. We denote by $K(\bar{V})$ the canonical bundle over $\bar{V}$.

Let $f: \boldsymbol{C}^{n} \rightarrow \bar{V}$ be a meromorphic mapping and $I(f)$ the indeterminancy locus of $f$. Then $f$ is holomorphic in $C^{n}-I(f)$. Define 
$\operatorname{rank} f=\max \left\{\operatorname{rank} d f(z) ; z \in \boldsymbol{C}^{n}-I(f)\right\}$.

We denote by $\operatorname{Mer*}\left(\boldsymbol{C}^{n}, \bar{V}\right)$ be the set of all meromorphic mappings $f: \boldsymbol{C}^{n} \rightarrow \bar{V}$ of maximal rank (i. e., $\operatorname{rank} f=\min \{m, n\})$ such that $f\left(\boldsymbol{C}^{n}\right) \cap V \neq \varnothing$.

Let $A(r)$ and $B(r)$ be real functions defined on $[1,+\infty)$. We write

$$
A(r) \leqq B(r) \|_{E}
$$

if $E \subset[1,+\infty)$ is a Borel subset with finite measure and if $A(r) \leqq B(r)$ for $r \in$ $[1,+\infty)-E$. We set

$$
\log ^{+} s=\log \max \{1, s\} \quad \text { for } s \in \boldsymbol{R} .
$$

Theorem (2.2). Let $1 \leqq n<m$ and $f \in \operatorname{Mer} *\left(\boldsymbol{C}^{n}, \bar{V}\right)$. Then we have

$$
\gamma\left\{T_{f}(r,[D])+T_{f}(r, K(\bar{V}))\right\} \leqq N\left(r, \operatorname{Supp} f^{*} D\right)+S(r),
$$

where for any $\varepsilon>0$,

$$
S(r)=n(1+\varepsilon)^{2} \log ^{+} T_{f}(r,[D])+\varepsilon n(2 n-1) \log r \|_{E(\varepsilon)} .
$$

In the case of $n \geqq m$, we have a similar but better estimate :

ThEOREM (2.5). Let $n \geqq m \geqq 1$ and $f \in \operatorname{Mer}^{*}\left(\boldsymbol{C}^{n}, \bar{V}\right)$. Then

$$
T_{f}(r,[D])+T_{f}(r, K(\bar{V})) \leqq N\left(r, \operatorname{Supp} f^{*} D\right)+S(r),
$$

where $S(r)$ is a small term as in (2.4).

Remark. See Sakai [18] for a more general theorem for equidimensional holomorphic mappings with maximal rank. The method we are going to use is different from his.

We will give the proofs of Theorems (2.2) and (2.5) step by step in what follows. First we assume $n<m$.

Let $f \in \operatorname{Mer} *\left(C^{n}, \bar{V}\right)$ and $\omega$ be the Kähler form associated with $g$. Since $V$ is complete hyperbolic, $f$ is holomorphic in $C^{n}-f^{-1} D$ and $f^{-1} D$ is a hypersurface. Put

$$
E=\left\{z \in C^{n} ; \operatorname{rank} d f(z)<n\right\} \cup f^{-1} D
$$

and $U=C^{n}-E$. Then $E$ is a thin analytic subset of $\boldsymbol{C}^{n}$. We put

$$
\left(f^{*} \omega\right)^{n}=\xi \alpha^{n}
$$

on $U$. First we show that

$$
\frac{\gamma}{\pi} f^{*} \omega \leqq d d^{c} \log \xi \quad \text { on } U \text {. }
$$

Since $f \mid U: U \rightarrow V$ is an immersion, $U$ may be considered as a locally closed 
complex submanifold of $\mathscr{D}$. Thus we have $(2.8)$ by the following lemma.

LEMMA (2.9). Let $N$ be an n-dimensional submanifold of $\mathscr{D}$ with inclusion mapping $\iota: N \rightarrow \mathscr{D}$. Let $\operatorname{Ric}_{N}(X, Y)$ the Ricci curvature of $\iota^{*} g$. Then

$$
\operatorname{Ric}_{N}(X, X) \leqq-\gamma .
$$

Proof. Let $H_{N}(X, Y)$ be the holomorphic bisectional curvature of $\iota^{*} g$. By [4], we see $H_{N}(X, Y) \leqq H(X, Y) \leqq 0$. Let $X \in \boldsymbol{T}_{p}(N)$ be a unit holomorphic tangent vector. Take an orthonormal basis $\left\{e_{1}, \cdots, e_{m}\right\}$ of $\boldsymbol{T}_{p}(N)$ with $e_{1}=X$. Then by [4], p. 226, we see that

$$
\begin{aligned}
\operatorname{Ric}_{N}(X, X) & =\sum_{\jmath=1}^{n} H_{N}\left(X, e_{\jmath}\right) \leqq \sum_{\jmath=1}^{n} H\left(X, e_{\jmath}\right) \\
& \leqq H\left(e_{1}, e_{1}\right) \leqq-\gamma
\end{aligned}
$$

This completes the proof of Lemma 2.9. Q.E.D.

LEMMA (2.10). Let the notation be as above.

(i) $f^{*} \omega$ is locally integrable on $\boldsymbol{C}^{n}$.

(ii) $\log \xi$ is locally written as the difference of two plurisubharmonic functions.

Proof. (i) It suffices to show that the local integrability of $f^{*} \omega$ around a point $p \in E$. Take a neighborhood $W$ of $p$ and a proper modification $\pi:\left(\widetilde{W}, \widetilde{E}^{\prime}\right)$ $\rightarrow\left(W, E^{\prime}\right)$ with $E^{\prime}=W \cap E$ satisfying the following:

(a) There exists a holomorphic mapping $\tilde{f}: \widetilde{W} \rightarrow \bar{V}$ such that $\tilde{f}=f \circ \pi$.

(b) $\widetilde{W}$ is smooth and $\left.\pi\right|_{\tilde{W}-\widetilde{E}^{\prime}}: \widetilde{W}-\widetilde{E}^{\prime} \rightarrow W-E^{\prime}$ is biholomorphic.

(c) $\tilde{E}^{\prime}$ is a hypersurface with only normal crossings.

Then it suffices to show the local integrability of $\pi^{*} f^{*} \omega=\tilde{f}^{*} \omega$ around a point $\tilde{p} \in \tilde{E}^{\prime}$. Take a holomorphic local cordinate system $\left(w_{1}, \cdots, w_{m}\right)$ around $\tilde{p}$ such that $\tilde{p}=(0, \cdots, 0)$ and $\widetilde{E}^{\prime}$ is locally defined by

$$
w_{1} \cdots w_{k}=0 \text {. }
$$

We may assume that the coordinates $w_{\text {, }}$ are defined in a neighborhood $\left\{\left|w_{j}\right| \leqq 1\right\}$. Let $\omega_{0}$ be the Kähler form defined by (1.3). Then by (2.1) and Schwarz's lemma, there exists a positive constant $C$ such that

$$
\tilde{f}^{*} \omega \leqq C \omega_{0} .
$$

Therefore $f^{*} \omega$ is locally integrable around $\tilde{p}$.

(ii) Taka a holomorphic function $F$ on $C^{n}$ so that $(F)=\operatorname{Supp} f^{*} D$. Then we see in the proof of (i) that $\log \left(\xi|F|^{2}\right)$ is locally bounded from above on $C^{n}$. On the other hand, we have 


$$
d d^{c} \log \left(\xi|F|^{2}\right) \geqq \frac{\gamma}{\pi} f^{*} \omega \geqq 0
$$

on $U$, so that $\log \left(\xi|F|^{2}\right)$ is a plurisubharmonic function on $C^{n}$. Since

we have (ii). Q.E.D.

$$
\log \xi=\log \left(\xi|F|^{2}\right)-\log |F|^{2},
$$

By Lemma (2.10), we see that $T_{f}(r, \omega)$ and

$$
\mu(r)=\frac{1}{2} \int_{S(r)} \log \xi \eta
$$

are defined.

LEMMA (2.12). We have

$$
\frac{\gamma}{\pi} T_{f}(r, \omega) \leqq N\left(r, \operatorname{Supp} f^{*} D\right)+\mu(r)+O(1) .
$$

Proof. Let $F$ be as in (2.11). Then by Lemma (1.6) and (2.8), we have

$$
d d^{c}\left[\log \left(\xi|F|^{2}\right)\right] \geqq \frac{\gamma}{\pi} f^{*} \omega .
$$

It follows from this, Lemmas (1.1) and (1.9) that

$$
\frac{\gamma}{\pi} T_{f}(r, \omega) \leqq N\left(r, \operatorname{Supp} f^{*} D\right)+\mu(r)+O(1) .
$$

Q.E.D.

LEMMA (2.13). We have

$$
\begin{aligned}
& T_{f}(r,[D])+T_{f}(r, K(\bar{V})) \\
& \leqq \frac{1}{\pi} T_{f}(r, \omega)+O\left(\log ^{+} T_{f}(r,[D])\right)+O(1) .
\end{aligned}
$$

Proof. Let $\Psi$ be a $C^{\infty}$-volume form on $\bar{V}$. By $[10$, p. 256], there exists a positive constant $C$ and a positive integer $l$ such that

$$
\Phi=\frac{\Psi}{|\sigma|^{2}\left(\log |\sigma|^{2}\right)^{2 l} \omega^{m}} \leqq C
$$

on $V$, where $|\cdot|$ is a hermitian metric in $[D]$ and $\sigma \in H^{\circ}(\bar{V},[D])$ with $|\sigma|<1$ and $(\sigma)=D$. Put

Then we have

$$
\zeta=f^{*} \Phi
$$




$$
\begin{aligned}
d d^{c} \log \zeta \geqq & f^{*} c_{1}(K(\bar{V}))-\frac{1}{\pi} f^{*} \omega \\
& +f^{*} c_{1}([D])-l d d^{c}\left[\log \left(\log \left|f^{*} \sigma\right|^{2}\right)^{2}\right] .
\end{aligned}
$$

on $U$. By making use of Lemma (1.1), (ii), Lemma (1.6) and the argument in the proof of Lemma (2.10), (i), we infer that

$$
\begin{aligned}
d d^{c}[\log \zeta] \geqq & f^{*} c_{1}(K(\bar{V}))-\frac{1}{\pi} f^{*} \omega \\
& +f^{*} c_{1}([D])-l d d^{c}\left[\log \left(\log \left|f^{*} \sigma\right|^{2}\right)^{2}\right] .
\end{aligned}
$$

Applying Lemma (1.9), we have

$$
\begin{aligned}
& T_{f}(r, K(\bar{V}))+T_{f}(r,[D]) \\
& \quad \leqq \frac{1}{\pi} T_{f}(r, \omega)+\frac{l}{2} \int_{S(r)} \log \left(\log \left|f^{*} \sigma\right|^{2}\right)^{2} \eta+O(1) \\
& \quad \leqq \frac{1}{\pi} T_{f}(r, \omega)+l \log ^{+} m_{f}(r, D)+O(1) \\
& \quad \leqq \frac{1}{\pi} T_{f}(r, \omega)+O\left(\log ^{+} T_{f}(r,[D])\right)+O(1) .
\end{aligned}
$$

Q.E.D.

By Lemmas (2.12) and (2.13), it remains to obtain an estimate of $\mu(r)$ for the proof of Theorem (2.2).

LEMMA (2.14). $\quad \xi^{1 / n} \alpha^{n} \leqq(1 / \pi) f^{*} \omega \wedge \alpha^{n-1}$.

Proof. Put

$$
f^{*} \omega=\frac{\sqrt{-1}}{2 \pi} \sum a_{j \bar{k}} d z_{j} \wedge d \bar{z}_{k}
$$

Then our claim follows from

$$
\begin{aligned}
n \xi^{1 / n} & =n\left(\operatorname{det}\left(a_{j \bar{k}}\right)\right)^{1 / n} \\
& \leqq \operatorname{trace}\left(a_{j \bar{k}}\right) .
\end{aligned}
$$

Q.E.D.

The following lemma is well known:

LEMMA (2.15). Let $h(r)(>0)$ be a monotone increasing function in $r \geqq 1$. Then $h(r)$ is differentiable at almost all $r$ and

$$
\frac{d h(r)}{d r} \leqq h(r)^{1+\varepsilon} \|_{E(s)}
$$


for any $\varepsilon>0$.

LEMMA (2.16). For $\varepsilon>0$, we have

$$
\mu(r) \leqq n(1+\varepsilon)^{2} \log ^{+} T_{f}(r,[D])+\varepsilon n(2 n-1) \log r \|_{E(\varepsilon)} .
$$

Proof. By making use of the concavity of logarithm function, we have

$$
\begin{aligned}
\mu(r) & =\frac{n}{2} \int_{S(r)} \log \xi^{1 / n} \eta \\
& \leqq \frac{n}{2} \log \left(\int_{S(r)} \xi^{1 / n} \eta\right) \\
& \leqq \frac{n}{2} \log \left(r^{1-2 n} \frac{1}{2 n} \frac{d}{d r} \int_{B(r)} \xi^{1 / n} \alpha^{n}\right) .
\end{aligned}
$$

Applying Lemma (2.15) to $h(r)=\int_{B(r)} \xi^{1 / n} \alpha^{n}$, we see that

$$
\mu(r) \leqq \frac{n}{2} \log \left(r^{1-2 n}\left(\int_{B(r)} \xi^{1 / n} \alpha^{n}\right)^{1+\varepsilon}\right) \|_{E_{1}(\varepsilon)} .
$$

It follows from Lemmas (2.14) and (2.15) that

$$
\begin{aligned}
\mu(r) & \leqq \frac{n}{2} \log \left(r^{1-2 n}\left(\int_{B(r)} \frac{1}{\pi} f^{*} \omega \wedge \alpha^{n-1}\right)^{1+\varepsilon}\right) \|_{E_{1}(\varepsilon)} \\
& =\frac{n}{2} \log \left(r^{1-2 n}\left(r^{2 n-1} \frac{d}{d r} T_{f}(r, \omega / \pi)\right)^{1+\varepsilon}\right) \|_{E_{1}(\varepsilon)} \\
& \leqq \frac{n}{2} \log \left(r^{1-2 n} r^{(2 n-1)(1+\varepsilon)}\left(T_{f}(r, \omega / \pi)\right)^{(1+\varepsilon)^{2}}\right) \|_{E_{2}(\varepsilon)},
\end{aligned}
$$

so that

$$
\mu(r) \leqq \frac{\varepsilon}{2} n(2 n-1) \log r+\frac{n(1+\varepsilon)^{2}}{2} \log ^{+} T_{f}(r, \omega / \pi) \|_{E_{2}(\varepsilon)} .
$$

We infer from Lemma (2.12) that

$$
\begin{aligned}
\log ^{+} T_{f}(r, \omega / \pi) & \leqq \log ^{+} \frac{1}{\gamma}\left\{N\left(r, \operatorname{Supp} f^{*} D\right)+\mu(r)\right\}+O(1) \\
& \leqq \log ^{+} N\left(r, f^{*} D\right)+\log ^{+} \mu(r)+O(1) \\
& \leqq \log ^{+} T_{f}(r,[D])+\log ^{+} \mu(r)+O(1)
\end{aligned}
$$

Thus we have by (2.17) and (2.18) 


$$
\begin{aligned}
\mu(r)-\frac{n(1+\varepsilon)^{2}}{2} \log ^{+} \mu(r) \leqq & \frac{n(1+\varepsilon)^{2}}{2} \log ^{+} T_{f}(r,[D]) \\
& +\frac{n(2 n-1) \varepsilon}{2} \log r+O(1) \|_{E_{2}(\varepsilon)} .
\end{aligned}
$$

Since

$$
\frac{n(1+\varepsilon)^{2}}{2} \log ^{+} \mu(r) \leqq \frac{\mu(r)}{2}
$$

for all large $\mu(r)$, we have

$$
\begin{aligned}
\mu(r) & \leqq n(1+\varepsilon)^{2} \log ^{+} T_{f}(r,[D])+n(2 n-1) \varepsilon \log r+O(1) \|_{E_{2}(\varepsilon)} \\
& \leqq n(1+\varepsilon)^{2} \log ^{+} T_{f}(r,[D])+n(2 n-1) \varepsilon \log r \|_{E_{3}(\varepsilon)} .
\end{aligned}
$$

Q.E.D.

Thus we have completed the proof of Theorem (2.2).

Proof of Theorem (2.5). In the case of $1 \leqq m \leqq n$, we may assume that

$$
\left(f^{*} \omega\right)^{m} \wedge\left(\bigwedge_{\jmath=1}^{n-m} \frac{\sqrt{-1}}{2 \pi} d z, \wedge d \bar{z}_{\jmath}\right) \neq 0 .
$$

We define a function $\xi$ by

$$
\xi \alpha^{n}=\left(f^{*} \omega\right)^{m} \wedge\left(\bigwedge_{\jmath=1}^{n-m} \frac{\sqrt{-1}}{2 \pi} d z_{\jmath} \wedge d \bar{z}_{\jmath}\right)
$$

(cf. (2.7)). Since Ricci $g=-1$, we immediately see that (2.8) holds with $\gamma=-1$. The remainder of the proof is the same as in that of Theorem (2.2) (cf. [5]).

\section{A defect relation and a ramification theorem for $f * D$}

In this section we prove some defect relations for $f \in \operatorname{Mer}^{*}\left(\boldsymbol{C}^{n}, \bar{V}\right)$, a degeneracy theorem and a ramification theorem. We keep the same notation as in $\S \S 1$ and 2 . We put

$$
\begin{aligned}
\theta_{0}=\theta_{0}(\mathscr{D}, \Gamma)= & \inf \{\mu \in \overline{\boldsymbol{Q}} ;|\nu(K(\bar{V})+\mu[D])| \text { has no base } \\
& \text { point in } \left.V \text { for some } \nu \in \boldsymbol{Z}^{+}\right\},
\end{aligned}
$$

where $|\nu(K(\bar{V})+\mu[D])|$ denotes the complete linear system of the line bundle $\nu(K(\bar{V})+\mu[D])$ with $\mu \nu \in Z$.

Remark. Note that $\theta_{0}<1$. In fact, it is known that for some $\nu \in \boldsymbol{Z}^{+}$, $|\nu K(\bar{V})+(\nu-1)[D]|$ has no base point in $V$. Unforunately, we can not determine the exact value of $\theta_{0}$, but in the case where $\mathscr{D}$ is a Siegel upper half space $\widetilde{S}_{B}$ and $\Gamma$ a Siegel modular group $\Gamma(k)$, we can give some estimate in 
terms of Siegel modular cusp forms (cf. [16]).

Theorem (3.2). Let $f \in \operatorname{Mer}^{*}\left(\boldsymbol{C}^{n}, \bar{V}\right)$.

(i) If $n<m$, then $\Theta_{f}(D) \leqq 1-\gamma\left(1-\theta_{0}\right)$.

(ii) If $n \geqq m$, then $\Theta_{f}(D) \leqq \theta_{0}$.

Proof. We assume that $n<m$. Since $V$ is hyperbolic, $f\left(\boldsymbol{C}^{n}\right) \cap D \neq \varnothing$, so that

$$
N\left(r, f^{*} D\right) \geqq C \log r
$$

for all large $r$, where $C$ is a positive constant. By Theorem (1.10), we have

$$
T_{f}(r,[D]) \geq C \log r+O(1) .
$$

We deduce from Theorem (2.2) that

$$
\Theta_{f}(D) \leqq 1-\gamma\left(1+\limsup _{r \rightarrow+\infty} \frac{T_{f}(r, K(\bar{V}))}{T_{f}(r,[D])}\right) .
$$

Take a rational number $\mu>\theta_{0}$. By the definition of $\theta_{0}$, there exists a global section $\tau \in H^{0}(\bar{V}, \nu(K(\bar{V})+\mu[D]))$ with $\nu \in Z^{+}$such that $f^{*} \tau \neq 0$. It follows from Theorem (1.10) that

Then

$$
\begin{aligned}
\nu\left(T_{f}(r, K(\bar{V}))+\mu T_{f}(r,[D])\right) & =N(r,(f * \tau))+m_{f}(r,(\tau))+O(1) \\
& \geqq O(1) .
\end{aligned}
$$

$$
\mu \geqq-\limsup _{r \rightarrow+\infty} \frac{T_{f}(r, K(\bar{V}))}{T_{f}(r,[D])},
$$

so that

$$
\theta_{0} \geqq-\limsup _{r \rightarrow+\infty} \frac{T_{f}(r, K(\bar{V}))}{T_{f}(r,[D])}
$$

We have by this and (3.3)

$$
\Theta_{f}(D) \leqq 1-\gamma\left(1-\theta_{0}\right) .
$$

In the case of $n \geqq m$, we use Theorem (2.5) instead of Theorem (2.2) in the above proof and obtain

$$
\Theta_{f}(D) \leqq \theta_{0}
$$

Q.E.D.

COROLlaRY (3.4) (cf. [11]). If $1 \leqq n<m$ and $\theta_{0}=\theta_{0}(\mathscr{D}, \Gamma)<1-1 / \gamma$, then the image of any non-constant holomorphic mapping from $\boldsymbol{C}$ into $\bar{V}$ must be disjoint from $V$. $\lambda$ if

Definition. We say that $f \in \operatorname{Mer} *\left(C^{n}, \bar{V}\right)$ ramifies over $D$ with order at least 


$$
f^{*} D \geqq \lambda \operatorname{Supp} f^{*} D \text {. }
$$

COROLlaRY (3.5) (Ramification Theorem). If $f \in \operatorname{Mer}^{*}\left(\boldsymbol{C}^{n}, \bar{V}\right)$ ramifies over $D$ with order at least $\lambda$, then

$$
\lambda \leqq \frac{1}{\gamma\left(1-\theta_{0}\right)}
$$

Remark. To see what Theorem (3.2) amounts to, we consider the case where $\mathscr{D}$ is the upper half plane $\boldsymbol{H}$. The assumption for $\Gamma$ to be neat and arithmetric is used only to ensure a good compactification $\overline{\Gamma \backslash \boldsymbol{H}}$ of $\Gamma \backslash \boldsymbol{H}$, and the argument works in the case where $\Gamma \subset P S L(2, \boldsymbol{R})$ is a Fuchsian group corresponding to a finite Riemann surface $S=\bar{S}-\left\{p_{i}\right\}_{i=1}^{d}$. Here $\bar{S}$ denotes a compact Riemann surface with genus $g$ and $p_{i}$ are distinct points of $\bar{S}$. In this case we have

$$
\begin{gathered}
r=1, \quad D=\sum_{\imath=1}^{d} p_{\imath}, \\
\theta_{0}=\frac{2-2 g}{d} . \\
\Theta_{f}(D)=1-\frac{1}{d} \limsup _{r \rightarrow+\infty} \frac{N\left(r, \operatorname{Supp} f^{*} D\right)}{T_{f}(r)},
\end{gathered}
$$

where $T_{f}(r)$ is the characteristic function with respect to the point bundle over $\bar{S}$. Therefore Theorem (3.2) yields the classical defect relation:

$$
\sum_{\imath=1}^{d} \Theta_{f}\left(p_{\imath}\right) \leqq 2-2 g \text {. }
$$

\section{The case of other domains than $C^{n}$}

(a) We consider a meromorphic mapping $f: X \rightarrow \bar{V}$ of maximal rank from a finitely sheeted covering space $X$ over $\boldsymbol{C}^{n}$ into $\bar{V}$ (cf. [12] and [14]). Let $R$ be the ramification divisor of $X \rightarrow C^{n}$ and assume that $n<m$. We define $T_{f}(r, \omega)$ and $N\left(r, \operatorname{Supp} f^{*} D\right)$ and etc. in the same way as in [12]. Then we have

$$
\gamma\left\{T_{f}(r,[D])+T_{f}(r, K(\bar{V}))\right\} \leqq N\left(r, \operatorname{Supp} f^{*} D\right)+N(r, R)+S(r),
$$

where $S(r)$ is the same as in (2.4). Note that $X \rightarrow C^{n}$ is algebraic if and only if

$$
N(r, R)=O(\log r) .
$$

Therefore, if $X$ is an affine algebraic, then the term $N(r, R)$ is included in $S(r)$.

(b) Let $\Delta$ be the unit disc $(|z|<1) \subset C$ and $\Delta^{*}=\Delta-\{0\}$. Let $f: \Delta^{*} \rightarrow \bar{V}$ be a non-constant holomorphic mapping such that $f\left(\Delta^{*}\right) \cap V \neq \varnothing$. We deal with the behavior of $f$ around the isolated singularity 0 . Changing the coordinate 
of $\Delta^{*}$, we write

$$
\Delta^{*}=\{w \in \boldsymbol{C} ;|w|>1\}
$$

and assume that $f$ is defined on

$$
\{w \in \boldsymbol{C} ;|w|>1-\delta\}
$$

for a small $\delta>0$. We define $T_{f}(r, \omega), N\left(r, \operatorname{Supp} f^{*} D\right)$ and etc. as in [13]. Then we have

$$
\gamma\left\{T_{f}(r,[D])+T_{f}(r, K(\bar{V})\} \leqq N\left(r, \operatorname{Supp} f^{*} D\right)+S(r),\right.
$$

where $S(r)$ is the same as in (2.4).

As an application, we give a Nevanlinna theoretic proof of the extension theorem due to [8] and [3].

Theorem (4.3). Let $f:\{0<|z|<1\} \rightarrow V$ be a holomorphic mapping. Then $f$ extends to a holomorphic mapping from $\{|z|<1\}$ to $\bar{V}$.

Proof. It follows from (4.2) that

$$
T_{f}(r,[D]+K(\bar{V}))=O(\log r),
$$

since $N\left(r, \operatorname{Supp} f^{*} D\right)=0$. This is sufficient to conclude the extension of $f$ (see [13]). Q.E.D.

Remark. See also [6], [9], [13] and [15] for related results.

\section{REFERENCES}

[1] A, Ash, D. Mumford, M. RAPOPORT AND Y. TAI, Smooth Compactification of Locally Symmetric Varieties, Math. Science Press, Brookline, 1975.

[2] K. Azukawa, Curvature operator of the Bergman metric on a homogeneous bounded domain, Tôhoku Math. J. 37 (1985), 197-223.

[3] A. BOREL, Some metric properties of arithmetic quotients of symmetric spaces and an extension theorem, J. Diff. Geometry 6 (1972), 543-560.

[4] S. I. Goldberg AND S. Kobay Ashi, On holomorphic bisectional curvature, J. Diff. Geometry 1 (1967), 225-233.

[5] P. A. GRIfFiths AND J. KING, Nevanlinna theory and holomorphic mappings between algebraic varieties, Acta Math. 130 (1973), 145-220.

[6] P. KIERnan, Extensions of holomorphic maps, Trans. Amer. Math. Soc. 172 (1972), 347-355.

[7] S. KobAYAshi, Hyperbolic Manifolds and Holomorphic Mappings, Marcel Dekker, New York, 1970.

[8] S. Kobayashi AND T. Ochiai, Satake compactification and the great Picard theorem, J. Math. Soc. Japan 23 (1971), 340-350.

[9] M. KwAck, Generalization of the big Picard theorem, Ann. Math. 90 (1969), 9-22. 
[10] D. Mumford, Hirzeburch's proportionality theorem in non-compact case, Invent. Math. 42 (1977), 239-272.

[11] A.M. NADEL, The non-existence of certain level structures on abelian varieties over complex function fields, Ann. Math. 129 (1989), 161-178.

[12] J. NoguchI, Meromorphic mappings of a covering space over $\boldsymbol{C}^{m}$ into a projective variety and defect relations, Hiroshima Math. J. 6 (1976), 265-280.

[13] J. Noguchi, Lemma on logarithmic derivatives and holomorphic curves in algebraic varieties, Nagoya Math. J. 83 (1981), 213-233.

[14] J. Noguchi, On the value distribution of meromorphic mappings of covering space over $\boldsymbol{C}^{m}$ into an algebraic variety, J. Math. Soc. Japan 37 (1985), 295-313.

[15] J. NoGUCHI, Moduli spaces of holomorphic mappings into hyperbolically imbedded complex spaces and locally symmetric spaces, Invent. Math. 93 (1988), 15-34.

[16] J. Noguchi, Moduli spaces of abelian varieties with level structure over function fields, International J. Math. 2 (1991), 183-194.

[17] J. Noguchi and T. OchiaI, Geometric Function Theory in Several Complex Variables, Translation of Math. Monographs, Vol. 80, Amer. Math. Soc., Providence, 1990.

[18] F. SAKaI, Defect relations and ramifications, Proc. Japan Acad. 50 (1974), 723728.

[19] B. Shiffman, Nevanlinna defect relations for singular divisors, Invent. Math. 31 (1975), 155-182.

[20] Y.T. SiU, Defect relations for holomorphic mappings between spaces of different dimensions, Duke Math. J. 55 (1987), 213-251.

[21] W. Stoll, Value distribution on parabolic spaces, Lect. Notes in Math., Vol.600, Springer-Verlag, Berlin-Heidelberg-New York, 1977.

Toshiba Co. R. and D. Center

1 Komukai Toshiba-cho, Saiwai-ku

KAWASAKI 210, JAPAN

AND

Department of Mathematics

Tokyo Institute of TeChNOLOGY

O'okayama, Meguro, Tokyo 152

JAPAN 